\title{
Vascularised free lymph node transfer - a procedure for secondary lymphoedema management in South Africa
}

\author{
D Liakos, ${ }^{1,2}$ C Sofianos, ${ }^{1}$ HN Sooka, ${ }^{1}$ RU Sheikh ${ }^{1}$ \\ ${ }^{1}$ Department of Plastic and Reconstructive Surgery, University of the Witwatersrand, Johannesburg, South Africa \\ ${ }^{2}$ Wits Donald Gordon Medical Centre, Eton Road, Johannesburg, South Africa
}

Corresponding author: Dr DimitriLiakos (plasticsurgery@drliakos.co.za)

\begin{abstract}
Summary
Lymphoedema is a condition in which impaired function of lymphatics results in poor lymphatic drainage and an accumulation of proteinaceous fluid in the interstitium. Lymphoedema has a broad clinical spectrum, and treatment options must be tailored to individual patients. Microsurgery is becoming popular as a surgical tool to manage refractory lymphoedema, and techniques include lymphaticovenous anastomosis(LVA) and vascularised lymph node transfer (VLNT). VLNT for treatment of lymphoedema has had promising outcomes thus far. We present a case report detailing the use of VLNT for treating post-surgical lymphoedema in the upper extremity.

Keywords: Lymphoedema, upper extremity, postsurgical, lymph node transfer, microsurgery
\end{abstract}

S Afr J Surg 2019;57(1)

http://dx.doi.org/10.17159/2078-5151/2019/v57n1a2785

\section{Introduction}

Lymphoedema is a condition in which impaired function of lymphatics results in poor lymphatic drainage and an accumulation of proteinaceous fluid in the interstitium. ${ }^{1}$ It affects over 250 million people worldwide, with filariasis being the commonest cause worldwide. ${ }^{2}$ In developed countries, iatrogenic causes such as irradiation and surgical therapy for malignancy are at the forefront in causing this disabling, life-long condition. Lymphoedema occurs secondary to mastectomy in up to $49 \%$ of patients, and up to $28 \%$ in lumpectomy, as well as in patients undergoing radiotherapy. ${ }^{3}$

Treatment of chronic lymphoedema is difficult as it lacks definitive, curative treatment and management is directed towards ameliorating symptoms. ${ }^{4}$ Conservative treatment measures have included complex decongestive therapy (CDT), manual lymphatic drainage, compression garments/ devices and meticulous skin care. ${ }^{5}$ Excisional procedures offer the benefit of size reduction in the lymphoedematous limb (up to $118 \%$ ), but at the cost of unaesthetic scarring and deformity as well as destruction of any remaining, viable lymphatics. ${ }^{6}$ Microsurgery is becoming popular as a surgical tool to manage refractory lymphoedema, and techniques include lymphaticovenous anastomosis (LVA) and vascularised lymph node transfer (VLNT).

VLNT was first demonstrated successfully in an animal model by $\mathrm{Shesol}^{7}$ and as a treatment for lymphoedema in 1990 after study in a canine model by Chen. ${ }^{8}$ This procedure brings vascularised lymph nodes into the lymphoedematous area, however lymphatic anastomoses are not done and lymphangiogenesis occurs spontaneously. ${ }^{9}$ This spontaneous mechanism has been attributed to the growth factors secreted by the transferred lymph nodes. ${ }^{10}$

\section{Case Report}

A 31-year-old female presented with a seven-year history of right upper extremity lymphoedema after excision of an axillary accessory breast at a regional hospital. She was referred to our quaternary institution for further management after a failure of six months of conservative therapy in the form of exercise and weight loss, skin care, manual lymphatic drainage and compression garment use. She had no comorbidities and was HIV negative. She reported new symptoms of pain in the affected limb, as well as heaviness which was worse at the end of the day. Examination revealed Stage II pitting lymphoedema. ${ }^{11}$ Table 1 shows her circumferential limb measurements at various levels. Figures 1 and 2 depict the right upper arm and the left (unaffected) upper extremity prior to surgery.

\begin{tabular}{lcc} 
Table 1. Circumferential limb measurements preoperatively \\
\hline Level & $\begin{array}{c}\text { Right upper } \\
\text { extremity } \\
\text { (lymphoedema) }\end{array}$ & $\begin{array}{c}\text { Left upper extremity } \\
\text { (no lymphoedema) }\end{array}$ \\
\hline Hand & $23.5 \mathrm{~cm}$ & $17 \mathrm{~cm}$ \\
$5 \mathrm{~cm}$ above wrist & $21 \mathrm{~cm}$ & $15.5 \mathrm{~cm}$
\end{tabular}




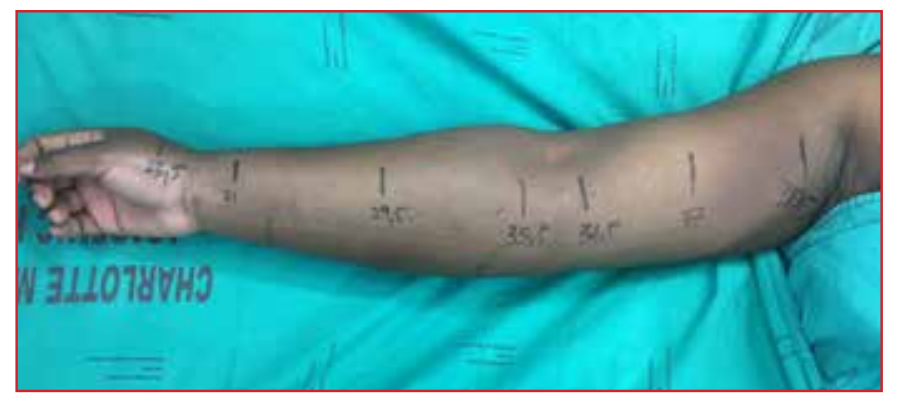

Figure 1: Lymphoedematous right upper arm

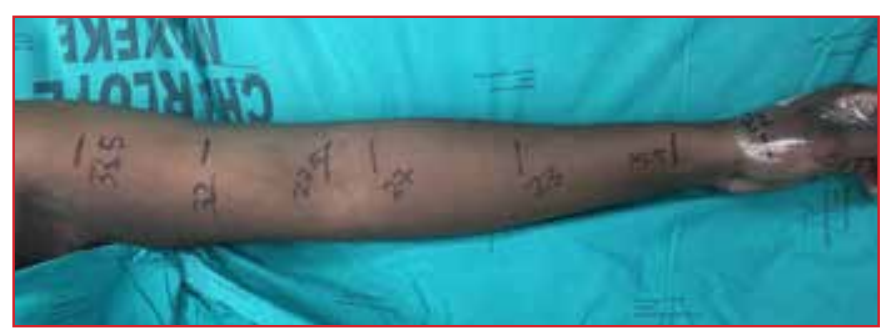

Figure 2: Normal left upper arm

$\begin{array}{lcc}15 \mathrm{~cm} \text { above wrist } & 29.5 \mathrm{~cm} & 23 \mathrm{~cm} \\ \text { Elbow } & 34.5 \mathrm{~cm} & 27.5 \mathrm{~cm} \\ 10 \mathrm{~cm} \text { above elbow } & 37 \mathrm{~cm} & 32 \mathrm{~cm}\end{array}$

\section{Management/Surgical Technique}

A surgical procedure was opted for in lieu of failure of conservative therapy. A VLNT was performed. The posterior triangle of the neck on the right was selected as the donor, and between four and five lymph nodes together with the

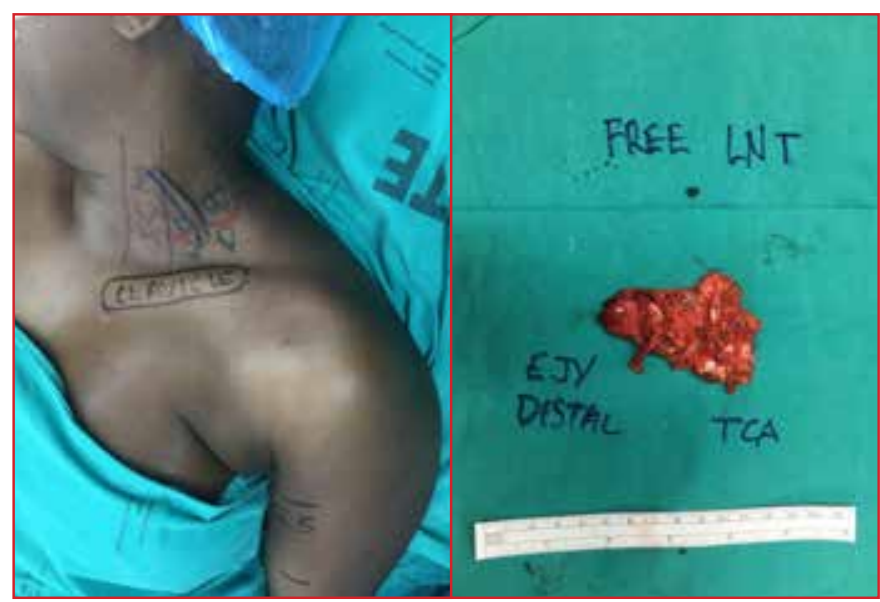

Figure 3A: Preoperative

Figure 3B: The harvested markings for supra-clavicular lymph node specimen, with lymph node harvest the transverse cervical artery (TCA) and external jugular vein $(E J V)$ as the pedicle

transverse cervical artery and vein, and external jugular vein (EJV) were raised in continuity. Preoperative surgical markings are illustrated in Figure 3. The harvest lymph node specimen with the relevant anatomy is depicted in Figure 4. In

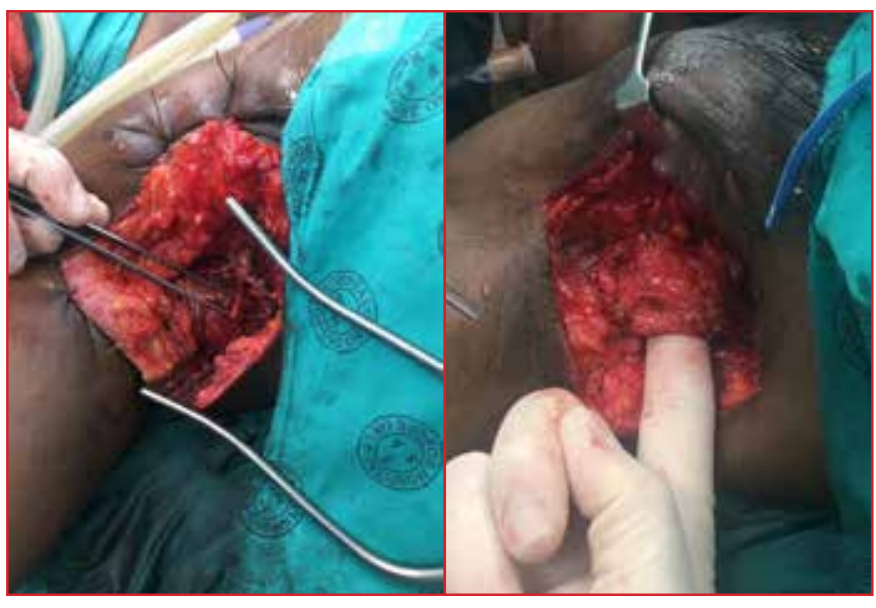

Figure 3C: The dissected axilla, with the prepared recipient thoracodorsal pedicle (forceps)

this case, the EJV was the dominant drainage vein.

The axillary recipient site (right side) was opened through the existing scar, and a wide dissection and excision of fibrotic scar tissue was performed and meticulous haemostasis obtained. The thoracodorsal vessels were identified and used as recipient vessels for the microvascular anastomosis (Figure 5).

A single stay suture was placed to retain the VLNT in its desired position (Figure 6). A suction drain was placed and the skin closed in layers. A splint preserving abduction of the shoulder was placed. The flap was noted to be perfusing well on-table intraoperatively.

The postoperative course was uneventful, and the drain was removed at day 2 postoperatively. The patient was discharged at day 5 postoperatively. She continued wearing the abduction thermoplastic splint at night for two weeks after which she resumed her physiotherapy regimen.

Postoperative limb circumference measurements were made at specific anatomic points as listed in Table 1, and measurements were made by the same surgeon to minimise inter-observer bias.

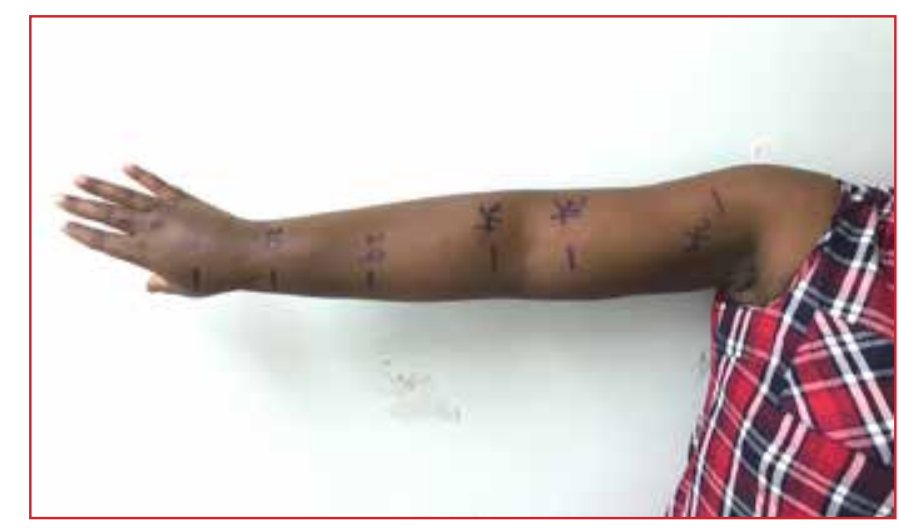

Figure 4: Six-month postoperative limb circumference measurements 


\section{Outcome}

Follow-up circumferential limb measurements at 6 months showed slight improvement in the affected limb as compared to preoperative measurements. It should be noted that it may take up to a year to see a clinical improvement. The majority of the improvement is noted in the proximal part of the limb as expected at six-month follow-up.

Table 2. Circumferential limb measurements of the right (lymphoedematous) upper extremity 6 months postoperatively

\begin{tabular}{lcc}
\hline Level & Preoperatively & $\begin{array}{c}\text { 6 months } \\
\text { postoperatively }\end{array}$ \\
\hline Hand & $23.5 \mathrm{~cm}$ & $23 \mathrm{~cm}$ \\
$5 \mathrm{~cm}$ above wrist & $21 \mathrm{~cm}$ & $20 \mathrm{~cm}$ \\
$15 \mathrm{~cm}$ above wrist & $29.5 \mathrm{~cm}$ & $29 \mathrm{~cm}$ \\
Elbow & $34.5 \mathrm{~cm}$ & $34 \mathrm{~cm}$ \\
$10 \mathrm{~cm}$ above elbow & $37 \mathrm{~cm}$ & $34 \mathrm{~cm}$
\end{tabular}

At six months postoperatively, the patient reported an improvement in symptoms, with less pain, less 'heaviness' of the limb, and improved softness of the soft tissue, especially in the proximal arm.

\section{Discussion}

Autologous VLNT is growing in popularity as a surgical treatment option for patients with lymphoedema. The procedure bridges the injured and interrupted lymphatic pathways, thereby re-establishing lymphatic flow. ${ }^{10}$ VLNT was introduced by O'Brien et al. and Chen et al. to treat obstructive lymphoedema in the canine model. ${ }^{8,12}$ The procedure was first described by Clodius et al. in a patient with lower leg lymphoedema, using a pedicled groin flap..$^{13}$ Becker et al. reported a series of 17 vascularised groin lymph node flaps to the axilla and seven to the elbow; forty-two percent of the cases returned to normal and fifty percent improved..$^{10}$ The procedure continues to grow in favour amongst lymphoedema surgeons as the primary treatment option for this debilitating condition.

Donor sites for VLNT consist of the lateral inguinal region, lateral thoracic flaps, or cervical flaps (submental or supraclavicular). Groin and axillary regions as a donor site have the advantage of a well-hidden scar, abundant soft tissue and, a consistent anatomy; however, there is a risk of donor site lymphoedema development. The supraclavicular and submental regions carry less risk of iatrogenic lymphoedema. ${ }^{3,14}$ Donor site selection may be driven by the need to avoid this morbidity.

Precise identification of the aetiology for lymphoedema is essential, and will guide the surgeon on placement of the VLNT. Preoperative imaging prior to surgery is critical to success of the VLNT.

There are three recipient sites available for vascularised groin lymph node flap transfer. In most cases of iatrogenic upper limb lymphoedema, the axillary area has been previously operated on and irradiated. Post radiation soft tissue changes make the dissection of recipient vessels more tedious. Both the elbow and the wrist are alternate areas for recipient vessel dissection, as they lie outside the radiated field. In our case, the axilla was chosen as no previous radiotherapy was performed. The choice between the elbow and wrist may depend on the patient's preferences regarding the aesthetic outcomes of the surgical site. Cheng et al. postulate that dependent areas of the limb, such as the elbow and wrist, may rely on gravity to enhance drainage of lymph from the surrounding interstitium into the transferred lymph node. ${ }^{15}$ Most importantly, it is difficult for the vascularised lymph nodes to drain the lymph against gravity, especially from the forearm and hand, which may consequently detract from clinical success of the transfer.

VLNT has been reported in clinical settings with or without a skin paddle, as was performed in this case. Both types of VLNT have demonstrated reasonable outcomes. The skin paddle can be useful as a monitor for checking the viability of the free flaps, but its function in the lymphoedematous limb is unknown.

Lymph nodes express vascular endothelial growth factor C3, which is responsible for lymphangiogenesis. ${ }^{9}$ Additionally, they serve to absorb excess extracellular fluid, in turn circulating this fluid into the venous system. The interaction between vascularised lymph node transfer and the lymphatic system in the recipient site is not well understood. There are two possibilities: by means of lymphovenous communication within the node or by means of the efferent lymphatic vessels from the lymph nodes. ${ }^{16}$ Lin et al. and Cheng et al. theorised that lymph nodes act as "lymph pumps," absorbing lymph fluid from the surrounding interstitium and transferring it into the venous circulation by means of lymphovenous communication within the nodes in the transferred flap..$^{15,17}$ This is an area which future research may elucidate in time to come.

\section{Conclusion}

Lymphoedema has a broad clinical spectrum, and treatment options must be tailored to individual patients. Vascularised lymph node transfer for treatment of lymphoedema has had promising outcomes thus far. The technique appears to be useful in cases of secondary lymphoedema, where an existing lymphatic network exists proximal and distal to the area of injury/obstruction. The complexity of the surgical technique and potential morbidity of VLNT may hinder its adoption amongst surgeons. However, the procedure is performed by all major microsurgical units around the world. The scientific medical literature has not reached a consensus on the mechanism of lymph node transfer, and selection criteria of patients have not been established. Further prospective clinical studies and basic scientific research are needed to evaluate the effectiveness of vascularised lymph node transfer for lymphoedema treatment.

\section{REFERENCES}

1. Kayiran O, De La Cruz C, Tane K, Soran A. Lymphedema: 
From diagnosis to treatment. Turk J Surg. 2017;33(2):51-7. Available from: http://dx.doi.org/10.5152/turkjsurg.2017.3870 [PMID:28740950]

2. Silva AK, Chang DW. Vascularized lymph node transfer and lymphovenous bypass: Novel treatment strategies for symptomatic lymphedema. J Surg Oncol. 2016;113(8):9329. Available from: http://dx.doi.org/10.1002/jso.24171 [PMID:26846735]

3. Raju A, Chang DW. Vascularized lymph node transfer for treatment of lymphedema: a comprehensive literature review. Ann Surg. 2015;261(5):1013-23. Available from: http://dx.doi. org/10.1097/SLA.0000000000000763 [PMID:24950271]

4. Viitanen TP, Visuri MT, Hartiala P, Maki MT, Seppanen MP, Suominen EA, et al. Lymphatic vessel function and lymphatic growth factor secretion after microvascular lymph node transfer in lymphedema patients. Plast Reconstr Surg Glob Open. 2013;1(2):1-9. Available from: http://dx.doi.org/10.1097/ GOX.0b013e318293a532 [PMID:25289206]

5. Scaglioni MF, Arvanitakis M, Chen YC, Giovanoli P, Chia-Shen Yang J, Chang EI. Comprehensive review of vascularized lymph node transfers for lymphedema: Outcomes and complications. Microsurgery. 2016. Available from: http://dx.doi.org/10.1002/ micr.30079 [PMID:27270748]

6. Cormier JN, Rourke L, Crosby M, Chang D, Armer J. The surgical treatment of lymphedema: a systematic review of the contemporary literature (2004-2010). Ann Surg Oncol. 2012;19(2):642-51. Available from: http://dx.doi.org/10.1245/ s10434-011-2017-4 [PMID:21863361]

7. Shesol BF, Nakashima R, Alavi A, Hamilton RW. Successful lymph node transplantation in rats, with restoration of lymphatic function. Plastic and reconstructive surgery. 1979;63(6):817-23. [PMID:441196]

8. Chen HC, O'Brien BM, Rogers IW, Pribaz JJ, Eaton CJ. Lymph node transfer for the treatment of obstructive lymphoedema in the canine model. Br J Plast Surg. 1990;43(5):578-86. [PMID:2224354]

9. Honkonen KM, Visuri MT, Tervala TV, Halonen PJ, Koivisto M, Lahteenvuo MT, et al. Lymph node transfer and perinodal lymphatic growth factor treatment for lymphedema. Ann Surg. 2013;257(5):961-7. Available from: http://dx.doi.org/10.1097/ SLA.0b013e31826ed043 [PMID:23013803]
10. Becker C, Vasile JV, Levine JL, Batista BN, Studinger RM, Chen CM, et al. Microlymphatic surgery for the treatment of iatrogenic lymphedema. Clin Plast Surg. 2012;39(4):385-98. Available from: http://dx.doi.org/10.1016/j.cps.2012.08.002 [PMID:23036289]

11. International Society of Lymphology. The diagnosis and treatment of peripheral lymphedema: 2013 Consensus Document of the International Society of Lymphology. Lymphology. 2013;46(1):1-11. [PMID:23930436]

12. O'Brien BM, Hickey MJ, Hurley JV, Dvir E, Khazanchi RK, Pederson WC, et al. Microsurgical transfer of the greater omentum in the treatment of canine obstructive lymphoedema. Br J Plast Surg. 1990;43(4):440-6. [PMID:2393770]

13. Clodius L, Smith PJ, Bruna J, Serafin D. The lymphatics of the groin flap. Ann Plast Surg. 1982;9(6):447-58. [PMID:7165238]

14. Cheng MH, Huang JJ, Nguyen DH, Saint-Cyr M, Zenn MR, Tan $\mathrm{BK}$, et al. A novel approach to the treatment of lower extremity lymphedema by transferring a vascularized submental lymph node flap to the ankle. Gynecol Oncol. 2012;126(1):93-8. Available from: http://dx.doi.org/10.1016/j.ygyno.2012.04.017 [PMID:22516659]

15. Poon Y, Wei CY. Vascularized groin lymph node flap transfer for postmastectomy upper limb lymphedema: flap anatomy, recipient sites, and outcomes. Plastic and reconstructive surgery. 2014;133(3):428e. Available from: http://dx.doi.org/10.1097/01. prs.0000438451.45227.b6 [PMID:24572896]

16. Ito R, Suami H. Overview of lymph node transfer for lymphedema treatment. Plastic and reconstructive surgery. 2014;134(3):548-56. Available from: http://dx.doi.org/10.1097/ PRS.0000000000000383 [PMID:25158711]

17. Lin $\mathrm{CH}$, Ali R, Chen SC, Wallace C, Chang YC, Chen HC, et al. Vascularized groin lymph node transfer using the wrist as a recipient site for management of postmastectomy upper extremity lymphedema. Plastic and reconstructive surgery. 2009;123(4):1265-75. Available from: http://dx.doi. org/10.1097/PRS.0b01e31819e6529 [PMID:19337095] 